\title{
Euclidean Axion Wormholes Have Multiple Negative Modes
}

\author{
Thomas Hertog, Brecht Truijen, and Thomas Van Riet \\ Institute for Theoretical Physics, KU Leuven, Celestijnenlaan 200D, B-3001 Leuven, Belgium
}

(Received 1 February 2019; revised manuscript received 28 May 2019; published 23 August 2019)

\begin{abstract}
We show that Euclidean axion wormholes in theories of gravity coupled to a single axion have several independent inhomogeneous perturbations that lower the Euclidean action. Our analysis relies on a judiciously chosen gauge-invariant variable which makes the negative mode structure about axion wormholes transparent. Perturbations lowering the action are concentrated in the neck region and exist for wormholes in flat space and in AdS. Their presence means axion wormholes are not relevant saddle points of the functional integral in quantum gravity. This resolves the paradoxes associated with these solutions from the viewpoint of AdS/CFT.
\end{abstract}

DOI: 10.1103/PhysRevLett.123.081302

Introduction.-Euclidean axion wormholes [1,2] are regular extrema of the action in semiclassical quantum gravity theories that connect two distant regions or even two disconnected asymptotic regions. Despite much work over many years, their status as saddle points of the functional integral remains unclear. Wormholes might be irrelevant, provide an imprint of other vacua of the landscape on our vacuum, or give rise to an intrinsic randomness of the observed constants of nature (see, e.g., [3-5]) with interesting phenomenological implications for cosmology and particle physics (see [6] for a recent review).

The weak gravity conjecture [7] lends some support to the physical relevance of wormholes, because its generalization to instantons implies the existence of superextremal instantons which, when sourced by axions, correspond to Euclidean axion wormholes [8-12].

On the other hand, wormholes connecting two asymptotic regions are deeply puzzling in the context of AdS/CFT $[13,14]$. From the field theory viewpoint the correlation functions across the two boundaries should factorize, while from the gravity point of view wormhole saddle point contributions would seem to introduce correlations between the two field theories.

One might have hoped that string theory would have avoided the paradoxes associated with Euclidean axion wormholes by not producing effective low-energy theories allowing wormhole solutions. After all, the existence of regular wormhole solutions depends delicately on the number of axions and dilatons in the theory and their couplings. However, that does not appear to be the case. In

Published by the American Physical Society under the terms of the Creative Commons Attribution 4.0 International license. Further distribution of this work must maintain attribution to the author(s) and the published article's title, journal citation, and DOI. Funded by SCOAP ${ }^{3}$. recent years, clean embeddings of axion wormholes in string theory have been given [14-16], further sharpening the paradox with AdS/CFT.

In this Letter, we provide evidence for a natural resolution of this paradox by analyzing the behavior of the action in the neighborhood of Euclidean axion wormholes (see [17-20] for earlier, inconclusive work on this). We work with four-dimensional theories of gravity coupled to a single axion and a cosmological constant $\Lambda \leq 0$. In these theories, we show that macroscopic axion wormholes which are everywhere in the semiclassical regime always have several independent inhomogeneous perturbations that lower the Euclidean action. This strongly suggests that axion wormholes are not relevant saddle points of the Euclidean functional integral.

To analyze whether there are physically meaningful perturbations that lower the action requires a careful choice of the perturbation variable for which the Euclidean action is well behaved and bounded below for normalized fluctuations. The theories we consider have a single physical scalar perturbation degree of freedom that is a combination of the axion and scalar metric perturbations about these wormholes. We identify the conjugate momentum $\Pi_{\mathcal{X}}$ of the gravitationally dressed gauge-invariant axion perturbation $\mathcal{X}$ as a suitable variable which makes the negative mode structure about wormholes transparent.

To derive the perturbation action, we follow the functional integral procedure developed in a series of papers [21-24] in which the degrees of freedom are identified in Lorentzian signature before the perturbation action is continued to the Euclidean. The boundary condition that the axion charge remains constant at the mouth of the wormhole implies vanishing Dirichlet boundary conditions on $\Pi_{\mathcal{X}}$. We find that this sets the homogeneous perturbation to zero, which, in turn, means the conformal factor problem does not interfere with our analysis. 
The action of perturbations that are inhomogeneous on three spheres at a constant scale factor is manifestly positive in the asymptotic regions of the wormhole, as one expects from the field theory limit. The kinetic term is positive everywhere, but the potential is negative (though bounded below) in the near-neck region. It is this feature, which is particular to axions, that gives rise to multiple independent perturbations concentrated in the near-neck region that lower the action.

The presence of negative modes often points towards the existence of a lower action saddle point. The fact that the perturbations lowering the action are inhomogeneous, however, suggests that in this case macroscopic wormholes fragment, ultimately breaking up the connection between the asymptotic regions.

Axions in Euclidean space.-Axionic scalars $\chi$ have a shift symmetry at the level of the classical action. The axion charge $Q$ under this symmetry is related to the radial derivative of the axion:

$$
Q \sim g^{r r} \sqrt{g} \partial_{r} \chi .
$$

Euclidean axion wormhole solutions are usually interpreted as instantons that violate axion charge conservation. This is because half wormholes describe the creation or absorption of baby universes, a process which from the point of view of the mother universe amounts to the loss or creation of axion charge.

Wormholes in this interpretation must be viewed as saddle points of the path integral in momentum space, since the boundary condition is that one fixes $Q$ at both wormhole ends [25]. This, as Eq. (1) shows, amounts to fixing the Euclidean axion momentum. Schematically, the axion charge transition amplitude reads

$$
K \equiv\left\langle\Pi_{F}|\exp (-H T)| \Pi_{I}\right\rangle
$$

where $|\Pi\rangle$ are axion momentum eigenstates, defined via a functional Fourier transform:

$$
|\Pi\rangle=\int d[\chi] e^{i \int_{\Sigma} \chi \Pi}|\chi\rangle .
$$

Here $\Pi$ is the radial component of a one-form that is orthogonal to the spacelike slice $\Sigma$. Hence, $|\Pi\rangle=|Q\rangle$, and momentum eigenstates are charge eigenstates.

It is well known (see, e.g., $[6,14,25,26])$ that axions have a kinetic term with the wrong sign in the Euclidean signature if one looks for saddle points of path integrals with fixed momentum boundary conditions. Equivalently, one can consider imaginary configurations in theories with normal sign kinetic terms as contributions to the transition amplitude (2). However, it is arguably more appealing to regard the Hodge dual formulation of the axion as fundamental, in which the axion enters as a $(D-1)$-form field strength $F$ and the action reads

$$
S=-\frac{1}{2 \kappa^{2}} \int \sqrt{|g|}\left(\mathcal{R}-\frac{1}{2} \frac{1}{(D-1) !} F^{2}-\Lambda\right),
$$

with $D$ the dimension. In this formulation, the axion has a standard kinetic term, and the saddle points are real solutions. Hodge duality transforms this action into the following:

$S=-\frac{1}{2 \kappa^{2}} \int \sqrt{|g|}\left(\mathcal{R}+\frac{1}{2} \nabla \chi \nabla \chi-\Lambda+\nabla(\chi \nabla \chi)\right)$.

This action has a wrong-sign kinetic term, at least when the axion is taken to be real. It also has an additional total derivative which provides an important contribution to the on-shell action.

Wormhole solutions. - We consider the simplest possible model with axionic wormholes, consisting of gravity coupled to a single axion and a negative cosmological constant $\Lambda=-\left(\alpha / l^{2}\right)$, where $l^{2}$ is the AdS radius and $\alpha=(D-1)(D-2)$. We write the metric of spherically symmetric wormhole solutions as

$$
d s^{2}=f(r)^{2} d r^{2}+a(r)^{2} d \Omega_{D-1}^{2},
$$

together with the axion profile $\chi(r)$. The axion equation of motion $\square \chi=0$ can be conveniently solved in terms of the radial harmonic function $h(r)$ defined as $d h=f a^{1-D} d r$. One finds that $\chi \propto h$ where the constant of proportionality squared,

$$
\left(\frac{d \chi}{d h}\right)^{2} \equiv c>0
$$

is precisely the axion charge $Q$ squared, i.e., the constant of motion associated with the shift symmetry of the axion. Using this, the Einstein equation reduces to a single firstorder equation for the scale factor [26,27]:

$$
\left(\frac{a^{\prime}}{f}\right)^{2}=1+\frac{a^{2}}{l^{2}}-\frac{c}{2 \alpha} a^{-2(D-2)}
$$

where $a^{\prime} \equiv \partial_{r} a$. Hence, a wormhole solution can be written as

$$
d s^{2}=\left(1+\frac{\tau^{2}}{l^{2}}-\frac{c}{2 \alpha} \tau^{-2(D-2)}\right)^{-1} d \tau^{2}+\tau^{2} d \Omega^{2},
$$

where $\tau$ is the scale factor $a$. The solutions asymptote to Euclidean AdS (EAdS) at large $\tau$ regardless of the value of $c$. When $c=0$, the solution is EAdS everywhere. In models (5) with a normal scalar rather than an axion, turning on the scalar yields $c<0$, which gives a singular solution that is a spikelike deformation of EAdS [26]. By contrast, for axions, $c>0$ and the solutions describe smooth wormholes with a minimum value $a(r=0)=$ $a_{0}$ of the scale factor given by 


$$
a_{0}=\left(\frac{c}{2 \alpha}\right)^{1 /(2 D-4)}+O\left(c / l^{2(D-2)}\right)^{2} .
$$

Macroscopic wormholes which are everywhere in the semiclassical regime must have $a_{0} \gg 1$ in Planck units and, hence, require $c \gg 1$. The regularity of the solutions at the neck means one does not expect these wormholes to carry a net axion charge. One can rather think of them as charge conduits [11], where both mouths carry opposite charges. The regularized Euclidean action of a full wormhole is

$$
S=\frac{\operatorname{Vol}\left(S^{D-1}\right)}{2 \kappa^{2}} \sqrt{\frac{2(D-1) \pi^{2}}{(D-2)}} \sqrt{c}+\mathcal{O}\left(a_{0} / l\right),
$$

where $\sqrt{c}$ is proportional to the quantized axion charge $Q$.

In theories with multiple axions and saxions (dilatons), the axion kinetic term is replaced by a sigma model, $G_{i j} \partial \phi^{i} \phi^{j}$, of indefinite signature. The instantons are then geodesic curves with $-c$ equal to the geodesic velocity squared [15].

Negative modes.-We now turn to the behavior of the action around the axionic Euclidean wormhole saddle points. We focus on wormholes in four dimensions, but our results likely generalize to other dimensions $D>2$. As discussed above, the action of quadratic perturbations of wormholes yields crucial input to elucidate the role-if any - of these wormholes in semiclassical quantum gravity. In particular, the existence and number of negative modes around the wormholes should, we believe, be a major consideration in their interpretation $[17,23,28]$.

To determine whether there are physically meaningful negative modes, it is imperative to work with well-behaved perturbation variables for which the Euclidean action is bounded below for normalized fluctuations. To this end, we follow the functional integral procedure developed in a series of papers [21-24] in which the physical degrees of freedom are carefully identified in Lorentzian signature before the perturbation action is continued to the Euclidean.

Perturbation action.-We write the line element of a general scalar perturbation of the Lorentzian continuation of the wormholes as follows:

$$
\begin{aligned}
d s^{2}= & b^{2}\left\{-(1+A)^{2} d \eta^{2}+\partial_{i} B d x^{i} d \eta\right. \\
& \left.+\left[(1-2 \psi) \gamma_{i j}+\partial_{i} \partial_{j} E\right] d x^{i} d x^{j}\right\},
\end{aligned}
$$

where we have adopted conformal gauge $f=a$, with $b$ the conformal scale factor and $\eta \in[0, \infty[$ the conformal time. The three-metric $\gamma_{i j}$ is the metric on $S^{3}$. Together with the fluctuation $\delta \chi$ of the scalar (axion) field, this results in five scalar perturbation fields in total.

Our starting point is the second-order Lorentzian action for a general linear scalar perturbation of this kind [22]. The constraints enforcing gauge invariance are imposed by introducing canonically conjugate momenta to rewrite the action in first-order form and then functionally integrating over the nondynamical fields. One then has some choice in deciding which linear combination of the remaining variables to use in order to describe the single physical scalar degree of freedom of the system. A suitable variable which makes the negative mode structure transparent is one whose Euclidean action is bounded below for normalized fluctuations around the backgrounds in question that obey the appropriate boundary conditions. This condition corresponds to having a positive kinetic term in the Euclidean action for all values of the Laplacian $\Delta$ on the three-sphere. It turns out that for axion wormholes a good physical choice of variable is the gauge-invariant combination $\mathcal{X}$ defined as

$$
\mathcal{X}=\psi+\frac{b^{\prime}}{b \chi^{\prime}} \delta \chi
$$

where the prime is now a derivative with respect to conformal time $\eta$. Substituting $\delta \chi$ for $\mathcal{X}$ in the action and performing the remaining functional integrals, we get the action of $\mathcal{X}$ [24].

It is convenient to decompose the perturbations in modes $\mathcal{X}_{n}$ multiplying spherical harmonics on the three-sphere where $n$ labels the eigenvalues $-n^{2}+1$ of $\Delta$, with $n>0$. The complete set of fluctuation modes divides into the $n=1$ mode, which is $O(4)$ invariant in the Euclidean region, and the $n>2$ modes, which describe inhomogeneous perturbations on the three-sphere. The $n=2$ mode is pure gauge. Continuing the action for $\mathcal{X}_{n}$ to Euclidean signature proceeds via $\rho=i \eta, \psi \rightarrow \psi$, and a Wick rotation of the axion field, this yields the results of Ref. [24] with $z$ changed to $i z$ :

$$
S_{2}=\frac{\operatorname{Vol}\left(S^{3}\right)}{\kappa^{2}} \int d \rho\left(A_{n} \dot{\mathcal{X}}_{n}^{2}-B_{n} \mathcal{X}_{n}^{2}\right),
$$

where a dot is a derivative with respect to Euclidean time $\rho$ and the functions $A_{n}$ and $B_{n}$ are given by

$$
\begin{gathered}
A_{n}=\frac{b^{2} z^{2}}{1+z^{2} /\left(4-n^{2}\right)}, \\
B_{n}=A_{n} \times\left(\frac{2}{1+z^{2} /\left(4-n^{2}\right)} \frac{\dot{z} b}{z \dot{b}}+5-n^{2}\right),
\end{gathered}
$$

respectively, with $z \equiv \dot{\chi} b / \dot{b}$ and $n \neq 2$. This variable $z$ differs by a factor $i$ from the corresponding variable in Ref. [24], which considers saddle points involving a scalar instead of an axion. The overall sign of the action (14) follows from the requirement that perturbations must be well behaved away from the neck region or, more generally, in the limit where gravity decouples [29].

We emphasized earlier that axionic wormholes should be viewed as saddle points of the Euclidean path integral in 
momentum space, with vanishing Dirichlet boundary conditions on the axion momentum at both wormhole ends. Our variable $\mathcal{X}$ is the gravitationally dressed axion. To complete the derivation of the perturbation action, we therefore express the action (14) in terms of the conjugate momentum $\Pi_{\mathcal{X}}^{n}$ :

$$
S_{2}=\frac{\operatorname{Vol}\left(S^{3}\right)}{\kappa^{2}} \int d \rho\left[-B_{n}^{-1}\left(\dot{\Pi}_{\mathcal{X}}^{n}\right)^{2}+A_{n}^{-1}\left(\Pi_{\mathcal{X}}^{n}\right)^{2}\right] .
$$

Stability analysis.-The kinetic term in this action is everywhere positive. The potential is bounded from below and negative only in the near-neck region. Moreover, the action has no divergences, and the gradient terms contribute positively. Hence, the momentum variable $\Pi_{\mathcal{X}}^{n}$ is a good physical choice of variable in which the negative mode structure around axionic wormholes can be analyzed.

In the absence of a cosmological constant, the coefficients $A_{n}$ and $B_{n}$ in the action (17) follow from Eqs. (7)-(9) that specify the wormhole backgrounds and read, respectively,

$$
\begin{aligned}
A_{n} & =\sqrt{\frac{|c|}{12}} \frac{\left(4-n^{2}\right) \cosh (2 \rho)}{3+\left(4-n^{2}\right) \sinh ^{2}(2 \rho)}, \\
B_{n}= & \sqrt{\frac{|c|}{12}} \frac{\left(4-n^{2}\right) \cosh (2 \rho)}{3+\left(4-n^{2}\right) \sinh ^{2}(2 \rho)} \\
& \times\left[\frac{-4\left(n^{2}-4\right) \cosh ^{2}(2 \rho)}{\left(n^{2}-4\right) \sinh ^{2}(2 \rho)-3}-n^{2}+5\right] .
\end{aligned}
$$

For the homogeneous $n=1$ perturbation, we have $B_{1}=0$, which implies $\dot{\Pi}_{\mathcal{X}}^{1}=0$ everywhere, consistent with earlier results [20] but in contrast with the non-gauge-invariant treatment in Ref. [17]. This means there is no homogeneous propagating degree of freedom. This is precisely what one expects, since the boundary conditions we selected on physical grounds fix the charge $Q$, leaving us with the Hamiltonian constraint (8) only. This also means we do not encounter a conformal factor problem with these boundary conditions, which resonates with Ref. [30].

Next, we consider the action of inhomogeneous $n>2$ perturbations. Figure 1 shows the functions $A_{n}$ and $B_{n}$ for $n=3$. Their behavior across the entire wormhole is qualitatively similar for all $n>2$. There is a singular point at $\rho=\rho^{*}$ where $A_{n}^{-1}$ and $B_{n}^{-1}$ both vanish. For $\rho>\rho^{*}$, in the asymptotic region of the wormhole where gravity is not important, the action is manifestly positive. By contrast, the potential is negative for $\rho<\rho^{*}$, in the neck region, leading to the possibility that perturbations confined to the neck region lower the action. The critical value $\rho^{*}$ decreases for increasing $n$. The Sturm-Liouville problem associated with the action (17) reads $\mathcal{O} \Pi_{\mathcal{X}}^{n}=\lambda \Pi_{\mathcal{X}}^{n}$ with the quadratic operator $\mathcal{O}$ given by

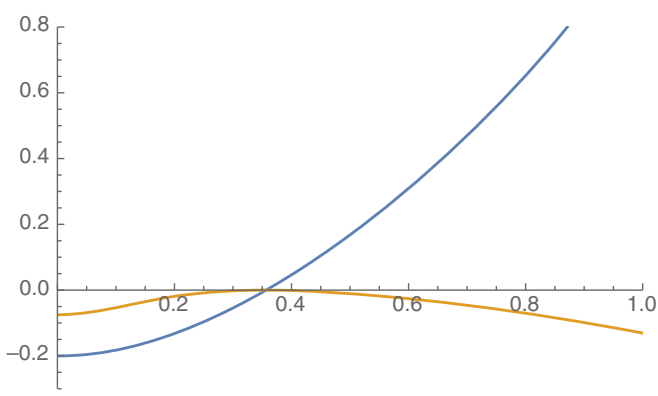

FIG. 1. The coefficients $A_{n}^{-1}$ (blue curve) and $B_{n}^{-1}$ (orange curve) entering in the action for perturbations about axion wormholes, shown here for $n=3$ (and with $c=1$ ).

$$
\mathcal{O}=B_{n}^{-1} \frac{d^{2}}{d \rho^{2}}+\dot{B}_{n}^{-1} \frac{d}{d \rho}+A_{n}^{-1}
$$

One can find negative modes either by an explicit diagonalization of this operator or by finding explicit test functions $\Pi_{\text {test }}^{n}$ that obey our boundary conditions and describe perturbations with negative action. Given that the potential term in the action is negative in the near-neck region, the latter method proves easier. Indeed, one readily sees that, e.g., the test function $\Pi_{\text {test }}^{n}=[\cosh (4 \rho)]^{-1}$ lowers the action, since $\left\langle\Pi_{\text {test }}^{n}|\mathcal{O}| \Pi_{\text {test }}^{n}\right\rangle<0$. Given the large degeneracy of the spherical harmonics on $S^{3}$, this means the action of macroscopic wormholes has multiple negative modes in the inhomogeneous sector. A single negative mode would have been consistent with an interpretation of the saddle points as mediating tunneling transitions. The negative determinant would in that case provide the imaginary piece of the Hamiltonian [28]. The presence of multiple negative modes means the wormhole saddle points do not contribute to any amplitude, in particular, not to the axion charge transition (2) of interest. All fluctuations lowering the action are concentrated around the wormhole neck, so it is really the nontrivial wormhole structure that is at the core of the instability. Although one can construct perturbations with negative action for all $n>2$, larger values of $n$ require those perturbations to die off faster.

When $\Lambda<0$, the metric of the wormholes is known only numerically in conformal gauge. A straightforward numerical analysis of the functions in the action (17) for nonzero $\Lambda$ shows that they exhibit a qualitatively similar behavior. Hence, also axionic wormholes connecting two asymptotically AdS regions have multiple negative modes. By contrast, a similar perturbation analysis applied to the class of instantons with a spike at the neck that are sourced by a dilaton, i.e., a scalar without shift symmetry which enters in the Euclidean theory with a normal kinetic term, shows that these have no negative modes.

Discussion.-We have calculated the second-order variation of the action of axion and scalar metric perturbations about macroscopic Euclidean axion wormholes in theories of gravity coupled to a single axion in flat space and in 
AdS. We have shown that there are multiple inhomogeneous small perturbations that lower the Euclidean action of these wormholes. This means that axion wormholes do not provide relevant saddle point contributions to the functional integral in semiclassical quantum gravity specifying axion charge transition amplitudes.

Our perturbation analysis is based on two crucial insights which resolve the ambiguities plaguing previous calculations and which make the negative mode structure about wormholes transparent. First, we have identified a gaugeinvariant variable, the conjugate momentum of the gravitationally dressed axion perturbation, for which the Euclidean action is everywhere well behaved and bounded below. Second, we have imposed Dirichlet boundary conditions on this to account for the fact that wormholes are saddle points of the functional integral in momentum space. It would be interesting to generalize our analysis to axion dilaton wormholes which in the Euclidean have kinetic terms of the form $-(\partial \phi)^{2}+e^{b \phi}(\partial \chi)^{2}$, where $\phi$ is the dilaton and $b$ a coupling constant. The addition of a dilaton allows for extremal D-instanton solutions, around which perturbations should have a manifestly positive quadratic action.

The presence of negative modes about a saddle point is usually a perturbative indication of the existence of a saddle point with lower action. In the case at hand, the fact that we find negative modes in the inhomogeneous sector suggests that macroscopic wormholes fragment. The lower action configuration one flows to might consist of an ensemble of microscopic "quantum wormholes" with unit charge. This is suggested by timelike $T$ duality which relates axion wormholes to superextremal objects with $Q>M$, which one expects fragmentate into superextremal particles of unit charge because the gravitational pull is weaker than electric repulsion.

But quantum wormholes have Planckian-sized necks and are not valid (regular) semiclassical saddle points. The above reasoning thus suggests that smooth geometric wormhole connections between distant regions are basically broken in gravitational theories with axions, thereby plausibly resolving the various paradoxes with AdS/CFT associated with these. The classically singular nature of microscopic quantum wormholes also means our stability analysis does not apply to these. Hence, our results leave open the possibility that unit charge wormholes enter as nonperturbative contributions to the axion potential, breaking the shift symmetry, with appealing implications for phenomenology.

We thank R. Alonso, B. Cottrell, A. Hebecker, K. M. Lee, M. Kramer, M. Montero, M. Trigiante, P. Soler, and A. Urbano for useful discussions and E. van der Woerd for initial collaboration. We also thank A. Hebecker, P. Soler, and E. Kiritsis for remarks on an earlier draft. The work of T. V.R. is supported by FWO Odysseus Grant No. G.0.E52.14N. The work of T. H. is supported in part by the National Science Foundation of Belgium (FWO) Grant No. G092617N, the C16/16/005 grant of the KU Leuven, and the European Research Council Grant No. ERC-2013-CoG 616732 HoloQosmos. B. T. acknowledges support by an FWO Ph.D. fellowship. We also acknowledge support from the COST Action MP1210 "The String Theory Universe."

[1] S. B. Giddings and A. Strominger, Axion induced topology change in quantum gravity and string theory, Nucl. Phys. B306, 890 (1988).

[2] G. V. Lavrelashvili, V. A. Rubakov, and P. G. Tinyakov, Disruption of quantum coherence upon a change in spatial topology in quantum gravity, Pis'ma Zh. Eksp. Teor. Fiz. 46, 134 (1987) [JETP Lett. 46, 167 (1987)].

[3] S. R. Coleman, Black holes as red herrings: Topological fluctuations and the loss of quantum coherence, Nucl. Phys. B307, 867 (1988).

[4] S. B. Giddings and A. Strominger, Loss of incoherence and determination of coupling constants in quantum gravity, Nucl. Phys. B307, 854 (1988).

[5] S. R. Coleman and K.-M. Lee, Wormholes made without massless matter fields, Nucl. Phys. B329, 387 (1990).

[6] A. Hebecker, T. Mikhail, and P. Soler, Euclidean wormholes, baby universes, and their impact on particle physics and cosmology, Front. Astron. Space Sci. 5, 35 (2018).

[7] N. Arkani-Hamed, L. Motl, A. Nicolis, and C. Vafa, The String landscape, black holes and gravity as the weakest force, J. High Energy Phys. 06 (2007) 060.

[8] T. Rudelius, Constraints on axion inflation from the weak gravity conjecture, J. Cosmol. Astropart. Phys. 09 (2015) 020.

[9] J. Brown, W. Cottrell, G. Shiu, and P. Soler, Fencing in the swampland: Quantum gravity constraints on large field inflation, J. High Energy Phys. 10 (2015) 023.

[10] M. Montero, A. M. Uranga, and I. Valenzuela, Transplanckian axions, J. High Energy Phys. 08 (2015) 032.

[11] B. Heidenreich, M. Reece, and T. Rudelius, Sharpening the weak gravity conjecture with dimensional reduction, J. High Energy Phys. 02 (2016) 140.

[12] A. Hebecker, P. Mangat, S. Theisen, and L. T. Witkowski, Can gravitational instantons really constrain axion inflation, J. High Energy Phys. 02 (2017) 097.

[13] J. M. Maldacena and L. Maoz, Wormholes in AdS, J. High Energy Phys. 02 (2004) 053.

[14] N. Arkani-Hamed, J. Orgera, and J. Polchinski, Euclidean wormholes in string theory, J. High Energy Phys. 12 (2007) 018.

[15] T. Hertog, M. Trigiante, and T. Van Riet, Axion wormholes in AdS compactifications, J. High Energy Phys. 06 (2017) 067.

[16] D. Ruggeri, M. Trigiante, and T. Van Riet, Instantons from geodesics in AdS moduli spaces, J. High Energy Phys. 03 (2018) 091.

[17] V. A. Rubakov and O. Yu. Shvedov, A negative mode about Euclidean wormhole, Phys. Lett. B 383, 258 (1996). 
[18] J. Y. Kim, H. W. Lee, and Y. S. Myung, Negative modes in the four-dimensional stringy wormholes, Phys. Rev. D 56, 6684 (1997).

[19] J. Y. Kim, Y.-b. Kim, and J. E. Hetrick, Classical stability of stringy wormholes in flat and AdS spaces, arXiv:hep-th/ 0301191.

[20] R. Alonso and A. Urbano, Wormholes and masses for Goldstone bosons, J. High Energy Phys. 02 (2019) 136.

[21] J. Garriga, X. Montes, M. Sasaki, and T. Tanaka, Canonical quantization of cosmological perturbations in the onebubble open universe, Nucl. Phys. B513, 343 (1998); Erratum, Nucl. Phys. B551, 511 (1999).

[22] S. Gratton and N. Turok, Cosmological perturbations from the no boundary Euclidean path integral, Phys. Rev. D 60, 123507 (1999).

[23] S. Gratton and N. Turok, Homogeneous modes of cosmological instantons, Phys. Rev. D 63, 123514 (2001).
[24] S. Gratton, A. Lewis, and N. Turok, Closed universes from cosmological instantons, Phys. Rev. D 65, 043513 (2002).

[25] C. P. Burgess and A. Kshirsagar, Wormholes and duality, Nucl. Phys. B324, 157 (1989).

[26] E. Bergshoeff, A. Collinucci, A. Ploegh, S. Vandoren, and T. Van Riet, Non-extremal D-instantons and the AdS/CFT correspondence, J. High Energy Phys. 01 (2006) 061.

[27] M. Gutperle and W. Sabra, Instantons and wormholes in Minkowski and (A)dS spaces, Nucl. Phys. B647, 344 (2002).

[28] S. R. Coleman, Quantum tunneling and negative eigenvalues, Nucl. Phys. B298, 178 (1988).

[29] We thank Arthur Hebecker and Pablo Soler for discussions of this point.

[30] E. Witten, A note on boundary conditions in euclidean gravity, arXiv:1805.11559. 